\title{
Destructive Reduction of TEX by Lithium-DFT Treatment
}

\section{Lemi Türker}

Department of Chemistry, Middle East Technical University, Üniversiteler, Eskişehir Yolu No: 1, 06800 Çankaya/Ankara, Turkey; e-mail: lturker@gmail.com; lturker@metu.edu.tr

\begin{abstract}
Interaction of lithium atom with TEX molecule which is a high density energetic material is considered within the restrictions of density functional theory at the level of UB3LYP/6-31++G(d,p). The results indicate that the lithium atom transfers an electron to TEX causing the rupture of one of $\mathrm{C}-\mathrm{N}$ bonds of the structure. Some geometrical and quantum chemical data have been collected and discussed. A plausible mechanism has been suggested for the destructive reduction of TEX molecule.
\end{abstract}

\section{Introduction}

Most of the metals (like $\mathrm{Li}, \mathrm{Na}$, etc.) fall into class of strong reducing agents delivering some of their valence electrons to organic molecules. The most encountered reductions of this type involve the electron transfer to $\pi$-electron systems.

Nitro compounds and their relatives are very readily reduced by many reagents. Depending on the acidic or basic conditions applied, various products from nitro compounds can be obtained. Nitro compounds are very versatile precursors for diverse functionalities. Nitramines are special type of nitro compounds.

The chemical degradation of nitramines has been of technical interest for years, since the nitro group has been used for protecting amines [1, 2]. Industrial applications of nitramine degradation have included the disposal of residual product and by-products from nitramine production, treatment of waste streams from the production processes, and, recently, the demilitarization of nitramine-based ordnance by denitration of the

Received: November 28, 2020; Accepted: January 2, 2021

Keywords and phrases: TEX, explosive, lithium, reduction, density functional.

Copyright (C) 2021 Lemi Türker. This is an open access article distributed under the Creative Commons Attribution License, which permits unrestricted use, distribution, and reproduction in any medium, provided the original work is properly cited. 
nitramine component [1-6]. Hydride reductions of various nitro aromatics including Nnitramines were reported [7].

A process of degrading nitramines by mixing the nitramine with an aqueous dispersion of metal powders and by heating the mixture is described [8] in which HMX and RDX are typical nitramines which are degraded. Metal powders which have been successfully used include aluminum and zinc. Best results have been obtained when an aqueous base is used in connection with the metal powder. Although, in the literature many articles exist about the reduction of nitrosamines [9, 10] very few reported for nitramines. Therefore, the interest and opportunity exist for the demonstration and development of a new, efficient and environmentally benign process for denitration of nitramine materials.

4,10-Dinitro-2,6,8,12-tetraoxa-4,10-diazawurtzitane (known as TEX) is as one of the novel energetic materials $[11,12]$. It is a nitramine having high density and very high detonation velocity [13-15]. In addition to those properties, TEX possesses extremely low sensitivity towards shock, friction and impulse [16]. It was first synthesized by Boyer and coworkers [12]. The synthesis of TEX involves the reaction of form amide with glyoxal and in a two-step synthesis involving a piperazine derivative as an intermediary product. TEX is a caged nitramine containing two embedded five-membered cyclic dietheric (also it can be considered as an acetal) structures resembling to 1,3-dioxalane.

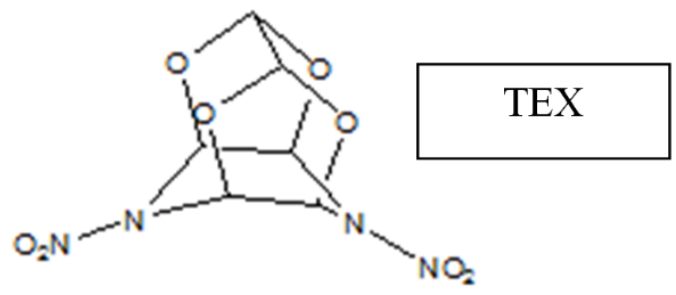

In the present study, interaction of lithium and TEX which is a nitramine type cagedhigh energy material, has been considered quantum chemically within the realm of density functional theory (DFT).

\section{Method of Calculations}

In the present study, firstly the initial structural optimizations of all the structures leading to energy minima have been achieved by using MM2 method followed by semiempirical PM3 self-consistent fields molecular orbital (SCF MO) method [17, 18] at the 
restricted level $[19,20]$. The subsequent optimizations were achieved at Hartree-Fock level using various basis sets hierarchically. Then, the structural optimizations were managed within the framework of density functional theory (DFT) [21, 22] at the levels of UB3LYP/6-31++G(d,p) [20, 23]. The exchange term of B3LYP consists of hybrid Hartree-Fock and local spin density (LSD) exchange functions with Becke's gradient correlation to LSD exchange $[22,24]$. The correlation term of B3LYP consists of the Vosko, Wilk, Nusair (VWN3) local correlation functional [25] and Lee, Yang, Parr (LYP) correlation correction functional [26]. Additionally, the vibrational analyses have been done. The total electronic energies are corrected for the zero point vibrational energy (ZPE). The normal mode analysis for each structure yielded no imaginary frequencies for the $3 N-6$ vibrational degrees of freedom, where $N$ is the number of atoms in the system. This indicates that the structure of each molecule corresponds to at least a local minimum on the potential energy surface. All these calculations were done by using the Spartan 06 package program [27].

\section{Results and Discussion}

Organolithium compounds are in general more convenient to handle and are also more soluble than all the organic compounds of the other alkaline metals. Because of that they find wide application in synthetic work. Also, the synthesis of organolithium compounds presents less difficulties in choosing the solvent [28].

TEX molecule has some susceptible sites for lithium interaction. The nitro groups have the prime possibility to undergo reaction with lithium. However, the attic or/and base ring (piperazine) hydrogens might show sufficient acidity to react with lithium atom because they are linked to electronegative atoms. Methalation reaction is suitable for the preparation of lithium derivative of comparatively acidic hydrogens.

Figure 1 shows the optimized structure of TEX+Li system in two different angles of view. As seen in the figure, lithium atom interacts with TEX molecule destructively causing the rupture of the $\mathrm{C}-\mathrm{N}$ linkage of the piperazine ring. Figure 2 shows the bond lengths/distances of the system. The broken $\mathrm{C}-\mathrm{N}$ bond yields the corresponding distance as $2.92 \AA$. The rest of the bond lengths are reasonable. The distances between the lithium atom to the oxygen and the nitrogen and carbon atoms of the broken bond are 2.01, 1.87, and $2.69 \AA$, respectively. 

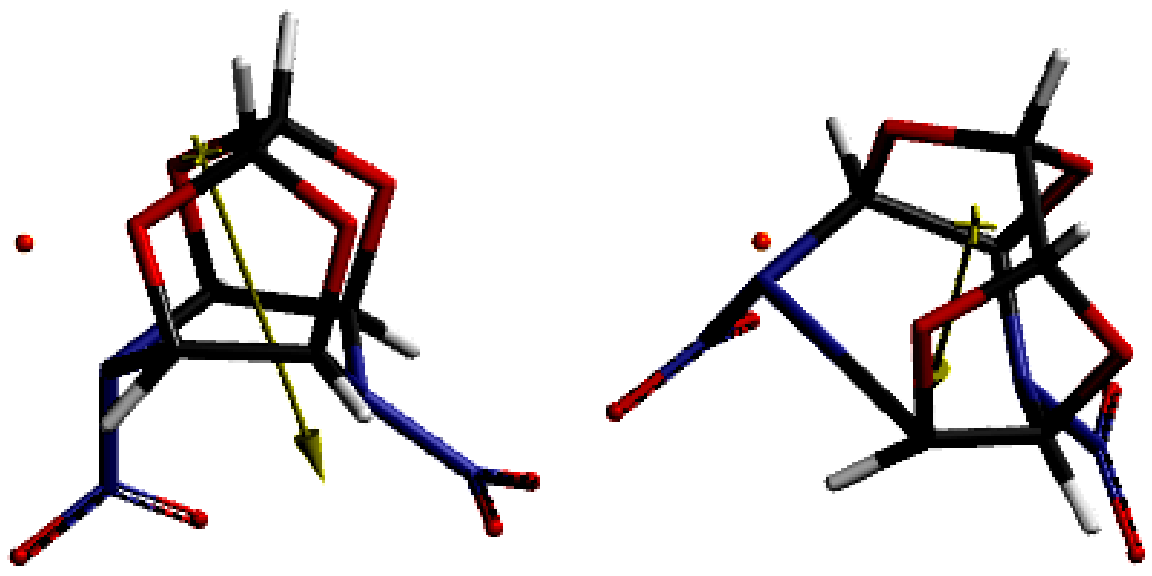

Figure 1. Optimized structure of TEX+Li system (two different views).

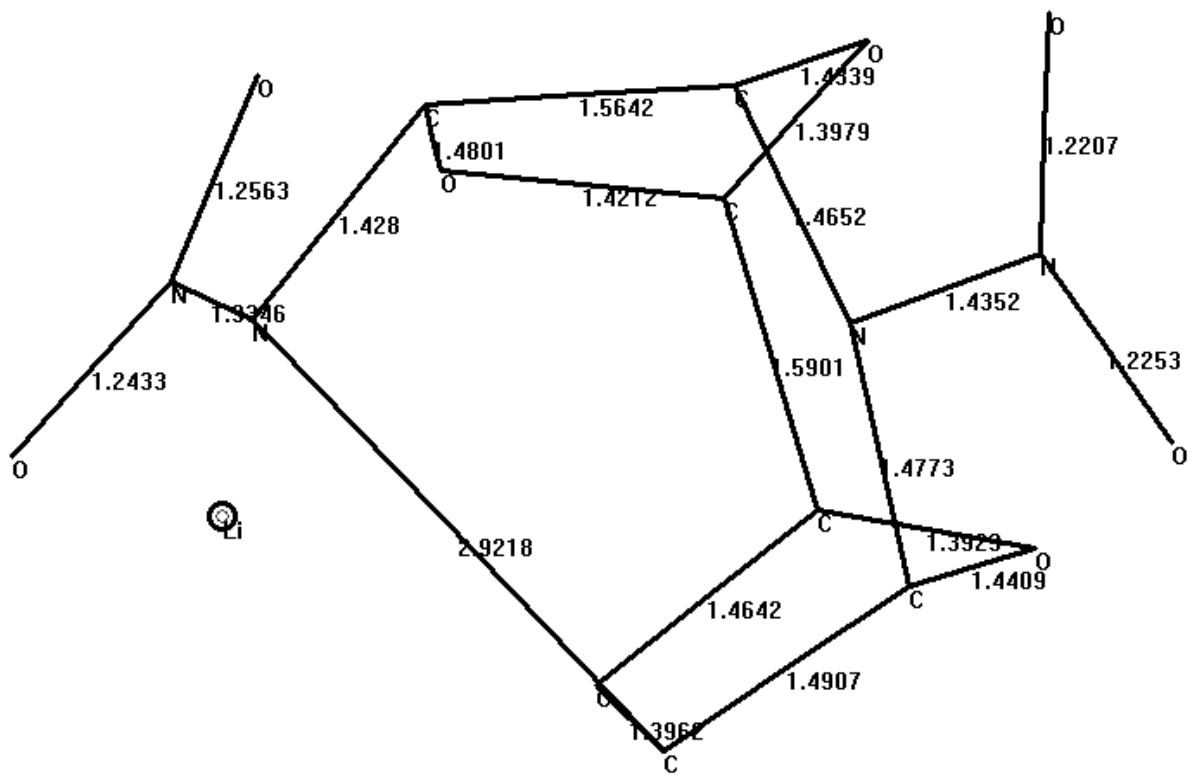

Figure 2. Bond lengths/distances of TEX+Li system (hydrogens omitted).

Figure 3 shows the electrostatic potential charges (ESP) on the atoms of the systems of TEX and TEX+Li. Note that the ESP charges are obtained by the program based on a numerical method that generates charges that reproduce the electrostatic potential field from the entire wavefunction [27]. 


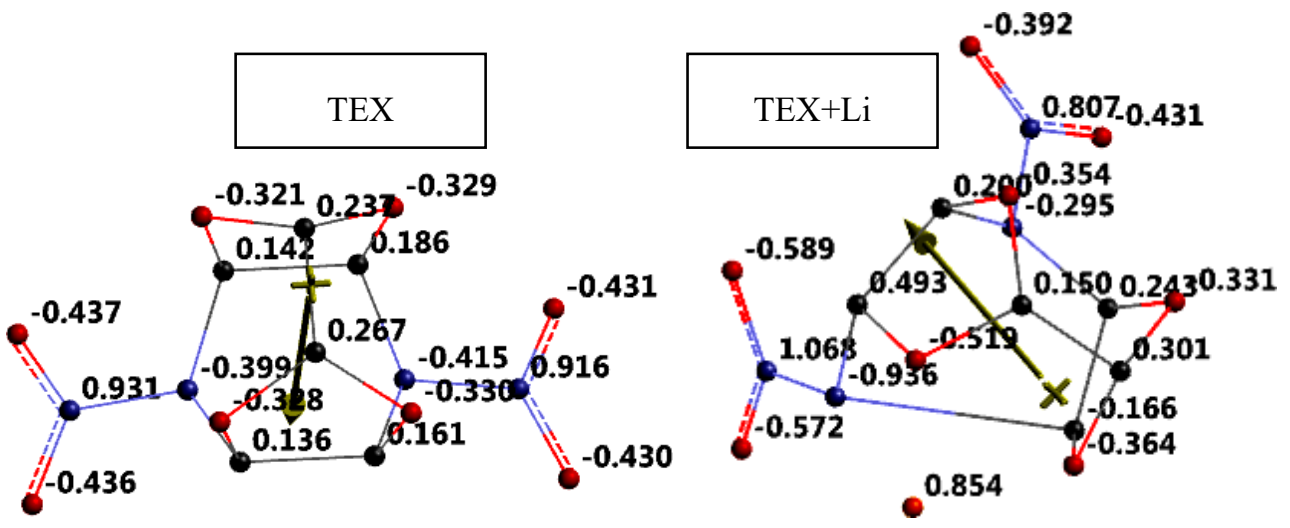

Figure 3. The ESP charges on the structures considered (hydrogens not shown).

The figure indicates that the lithium atom acquires some positive charge close to unity $(0.854 \mathrm{eu})$. The charges on the nitrogen and carbon atoms of the broken bond are -0.936 and $-0.166 \mathrm{eu}$, respectively. Figure 4 displays the electrostatic potential maps of TEX and TEX+Li systems. In the figure the blue/bluish and red/reddish regions show the positive and negative potential regions, respectively. In the TEX molecule the positive potential regions coincide with the attic and base regions whereas in the lithium composite a huge region around the lithium atom extending to the attic region exists.
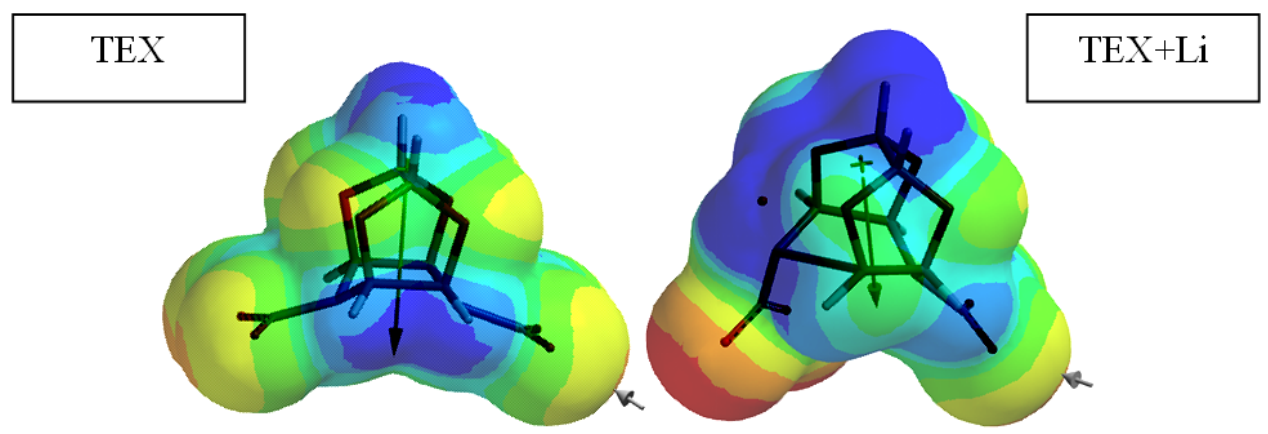

Figure 4. Electrostatic potential maps of the structures considered.

Figure 5 shows some of the molecular orbital energy levels of the systems considered. Note that lithium atom has an unpaired electron in its ground state electronic configuration. 

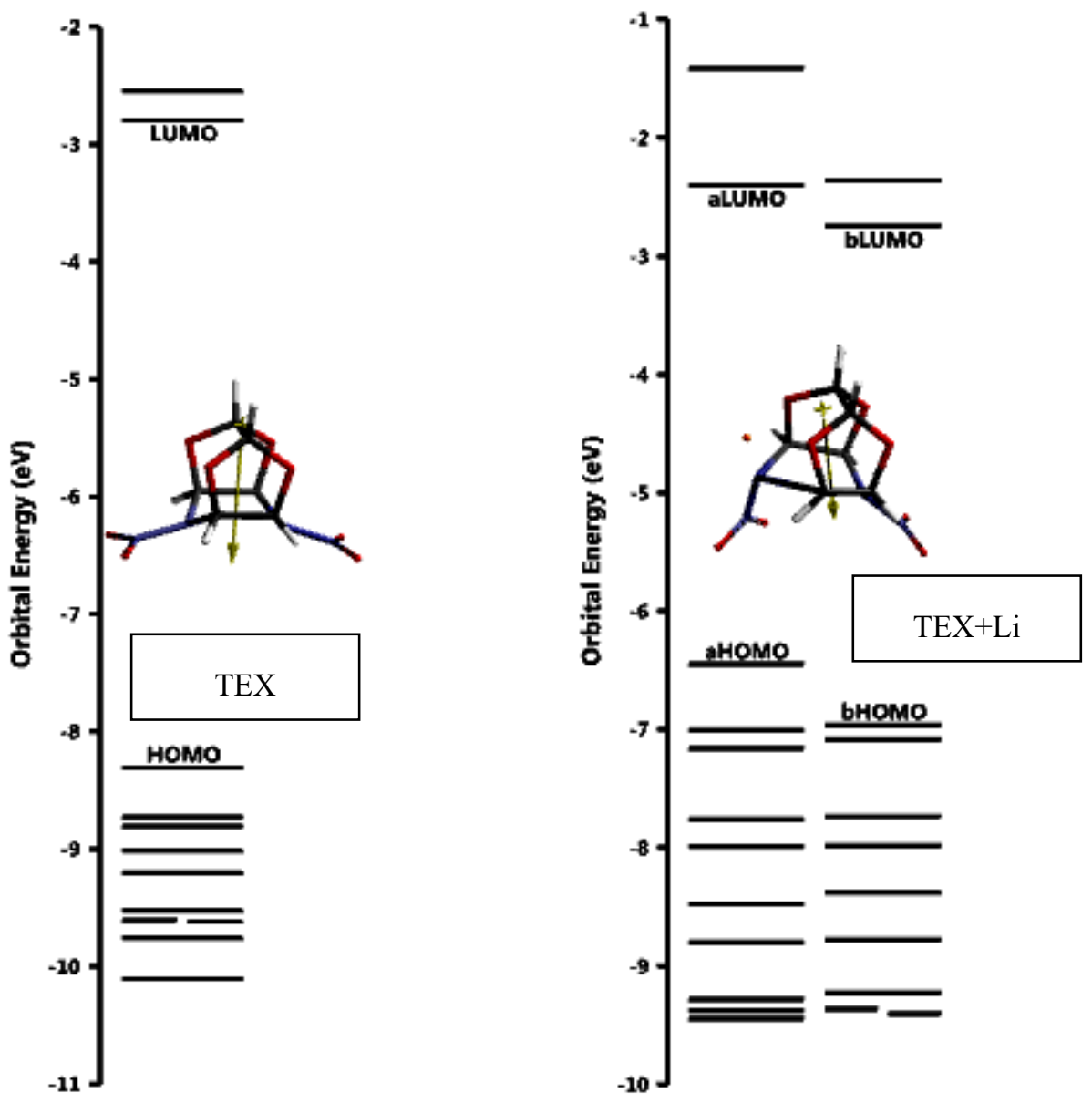

Figure 5. Some of the molecular orbital energy levels of the systems considered.

Therefore, the presently applied unrestricted level of calculation yields $\alpha$ - and $\beta$-type molecular orbitals which are represented as a- and b-types in the figure. As seen in the figure, the presence of lithium atom causes mainly the raise of the HOMO level up compared to the parent structure, TEX. The LUMO level is raised somewhat as well (see Table 1). All these are indication of transfer of some electron population to the organic component of the system. The data in Table 1 also indicate that the interfrontier molecular orbital energy gap $(\Delta \varepsilon)$ is much narrower in TEX+Li system as compared to the respective value of TEX molecule. 
Table 1. The HOMO, LUMO energies and $\Delta \varepsilon$ values of the structures considered.

\begin{tabular}{lccc}
\hline Structure & HOMO & LUMO & $\Delta \boldsymbol{\varepsilon}$ \\
\hline TEX & -801.49 & -269.52 & 531.97 \\
TEX+Li & -622.29 & -231.50 & 390.79 \\
\hline
\end{tabular}

Energies in $\mathrm{kJ} / \mathrm{mol}$.

Figure 6 shows the time-dependent density functional UV-VIS spectrum of TEX+Li system which covers a large domain in the spectrum.

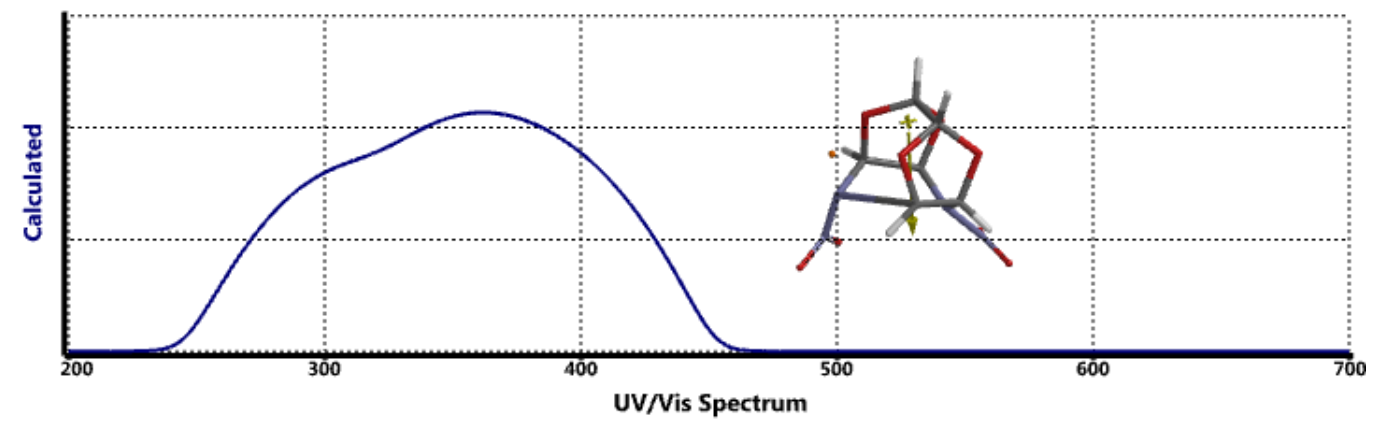

Figure 6. UV-VIS spectrum of TEX+Li system.

Figure 7 is the local ionization maps of the systems considered. In the local ionization potential map conventionally red regions on the density surface indicate areas from which electron removal is relatively easy, meaning that they are subject to electrophilic attack.
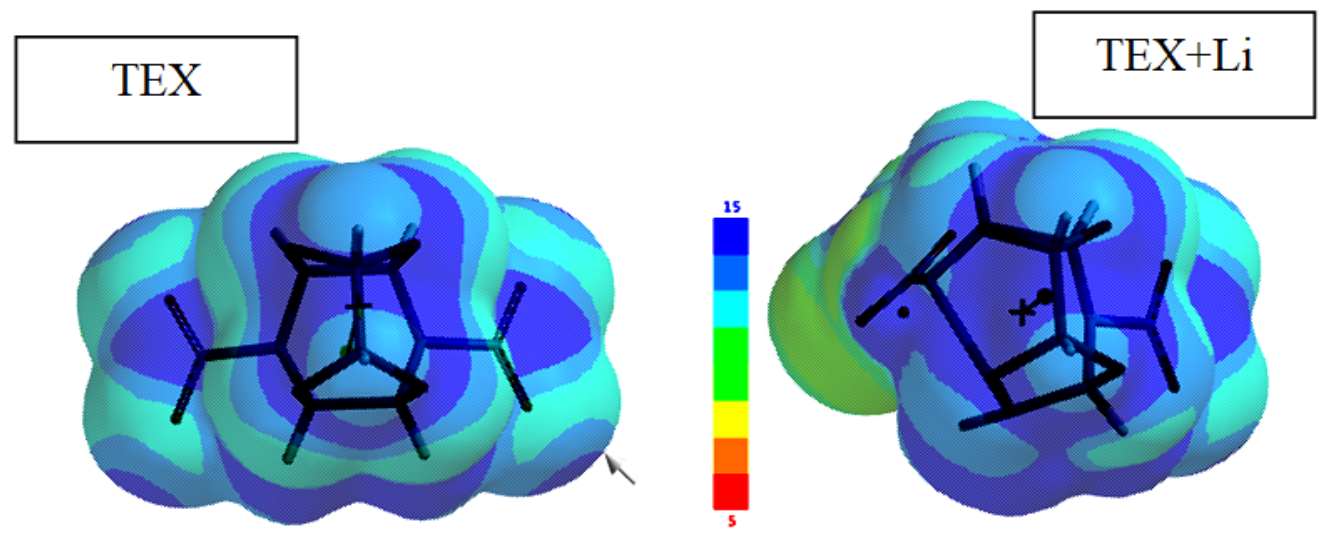

Figure 7. Local ionization maps of the systems considered.

Figure 8 is the LUMO map of TEX molecule. A LUMO map displays the absolute 
value of the LUMO on the electron density surface. The blue color stands for the maximum value of the LUMO and the color red, the minimum value and the yellowgreen regions are in between. Hence, a nucleophile attacks on the atom having the blue color.
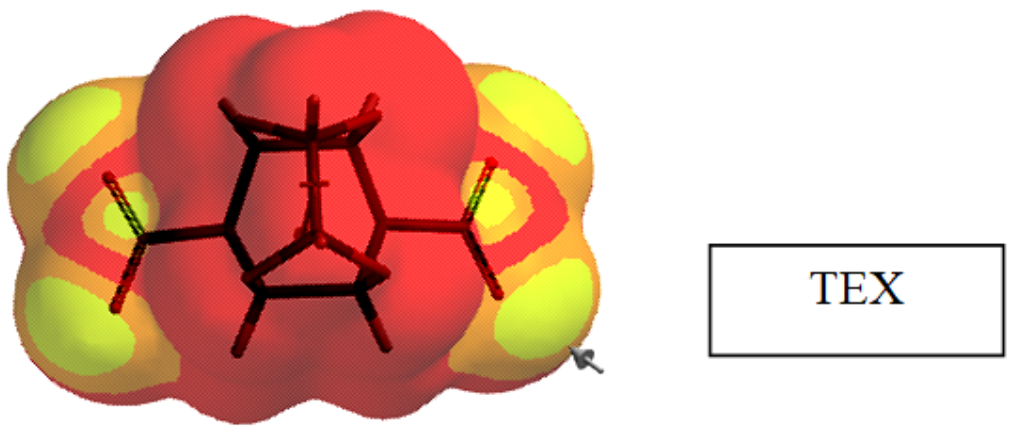

Figure 8. The LUMO map of TEX molecule.

Figure 9 displays the spin density map of TEX+Li system where the blue region indicates the site of the unpaired electron population.

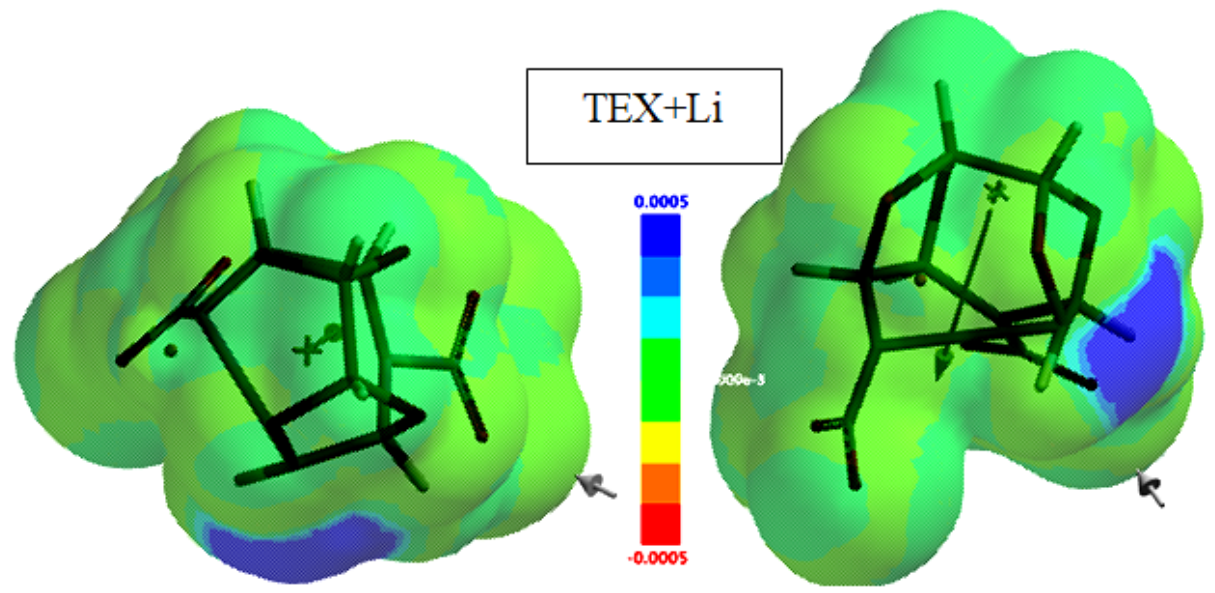

Figure 9. Spin density map of TEX+Li system.

In nitramines the electron withdrawing effect of nitro group is mainly inductive because in resonance structure-B two positive charges on adjacent nitrogens appear which is highly energetic and unfavorable situation. 

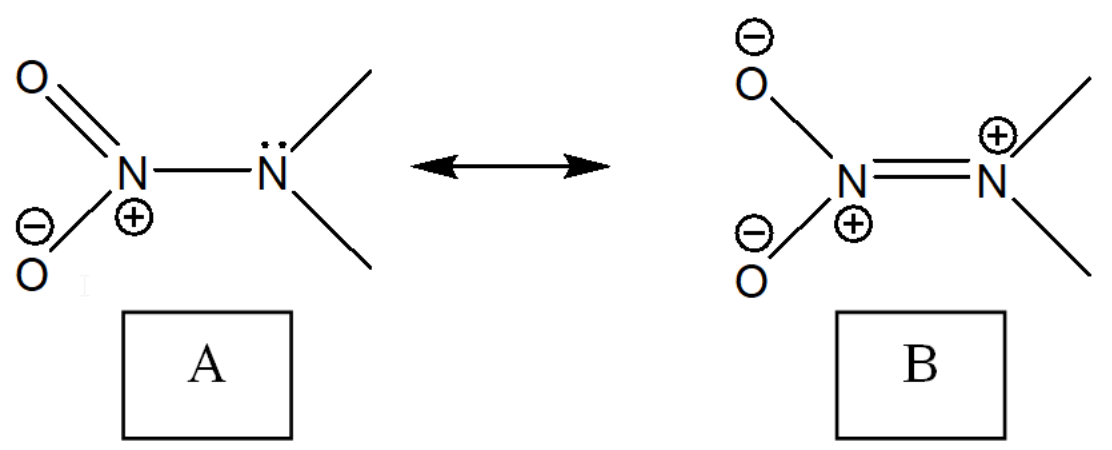

The electron from the lithium atom cannot be localized on the nitro group because this time electron-lone-pair repulsion develops between the nitramine nitrogen atoms. On the other hand, the carbon atom adjacent to the nitramine moiety is under the inductive electron demand of the neighboring nitrogen and oxygen atoms in the TEX structure. In the below scheme, structure-A possesses positive charge-lone-pair attractive interaction between the nitrogen atoms. In structure-B1 opposite charges are on the adjacent sites, therefore a more favorable situation arises compared to structure-A. Moreover, resonance structure- $C$ stabilizes the decomposed system in which favorable charge-charge and charge-lone-pair interactions exist. Note that the separate moieties B1 and B2 are linked to each other in the decomposed TEX molecule.

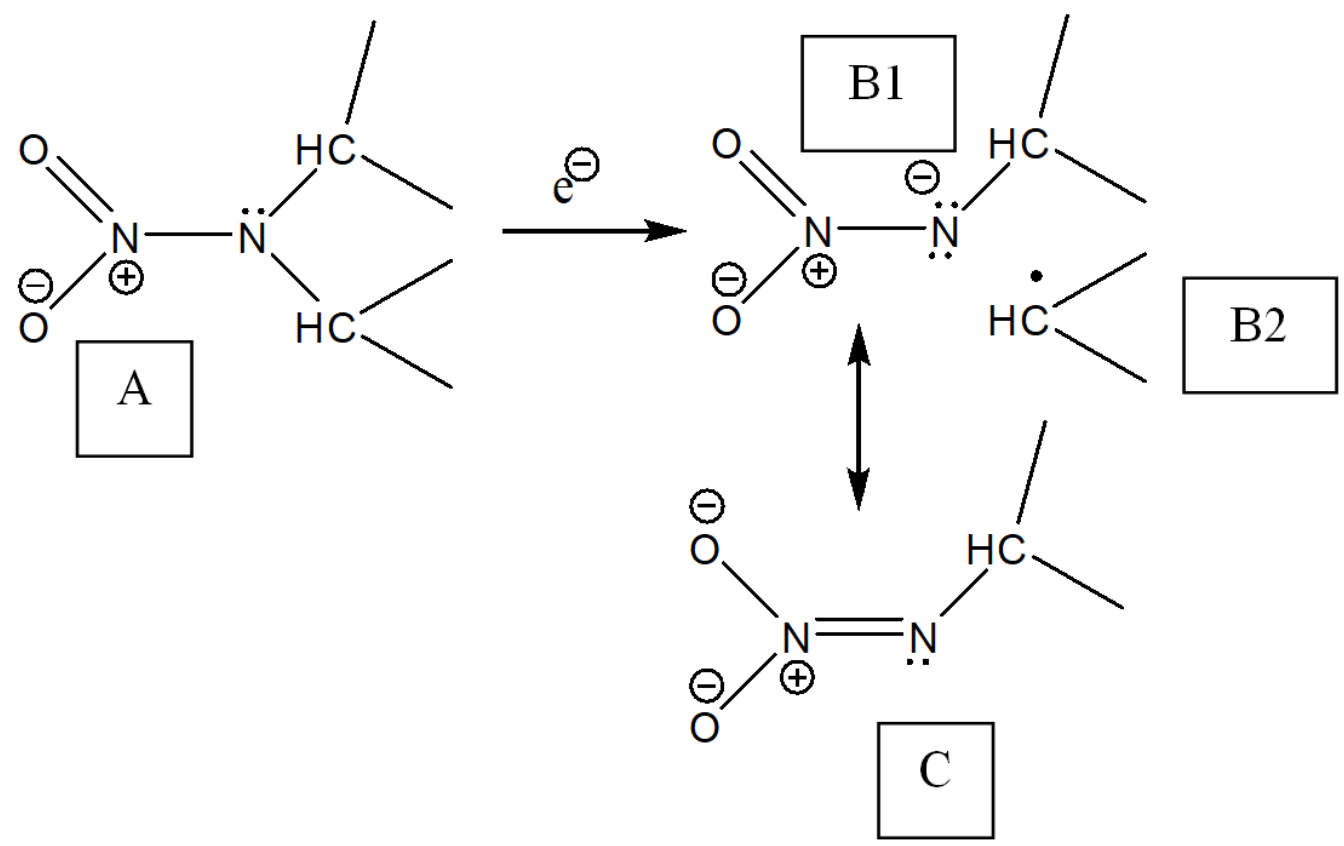


In Figure 9 unpaired electron density (blue region) is on the carbon atom indicated as $\mathrm{B} 2$ in the scheme.

\section{Conclusion}

The present study (within the constraints of the theory and the level of basis set used) indicates that the lithium atom transfers some electron population to TEX molecule causing its destructive reduction. The destruction is in the C-NNO2 bond, where the nitro group remains unaffected. The proposed mechanism of destruction involves a radical formation on the carbon atom as in accord with the spin density map obtained by calculation. In practice the radical formed may undergo some subsequent reactions which might be some potent cyclic/acyclic compounds.

\section{References}

[1] R.D. Chapman, R.A. O'Brien and P.A. Kondracki, N-Denitration of nitramines by dihydronicotinamides, Tetrahedron 52(29) (1996), 9655-9664. https://doi.org/10.1016/0040-4020(96)00502-9

[2] T.W. Greene and P.G.M. Wuts, Protective Groups in Organic Synthesis, 2nd ed., New York: Wiley, 1991, p. 374.

[3] C.L. Kitts, D.P. Cunnıngham and P.J. Unkefer, Isolation of three hexahydro-1,3,5trinitro-1,3,5-triazine-degrading species of the family enterobacteriaceae from nitramine explosive-contaminated soil, Applied and Environmental Microbiology 60(12) (1994), 4608-4611. https://doi.org/10.1128/aem.60.12.4608-4611.1994

[4] H. Nivinskas, J. Sarlauskas, Z. Anusevicius, H.S. Toogood, N.S. Scrutton and N. Cenas, Reduction of aliphatic nitroesters and N-nitramines by Enterobacter cloacae PB2 pentaerythritol tetranitrate reductase: quantitative structure-activity relationships, FEBS J. 275(24) (2008), 6192-203. https://doi.org/10.1111/j.1742-4658.2008.06744.x

[5] V.K. Balakrishnan, F. Monteil-Rivera, A. Halasz, A. Corbeanu and J. Hawari, Decomposition of the polycyclic nitramine explosive, CL-20, by Fe0, Environmental Science and Technology 38(24) (2004), 6861-6866. https://doi.org/10.1021/es049423h

[6] P. Armas, C.G. Francisco, R. Hernández and E. Suárez, Reduction of aliphatic nitramines. Approach to the synthesis of nitrosamines and amines, Tetrahedron Letters 27(27) (1986), 3195-3198. https://doi.org/10.1016/S0040-4039(00)84752-8

[7] H. Nivinskas, R.L. Koder, Ž. Anusevičius, J. Šarlauskas, A.F. Miller and N. Cenas, Quantitative structure-activity relationships in two electron reduction of nitroaromatic 
compounds by Enterobacter cloacae NAD(P)H:Nitroreductase, Archives of Biochemistry and Biophysics 385(1) (2001), 170-178. https://doi.org/10.1006/abbi.2000.2127

[8] L.F. Cannizzo and L.R. Huntsman, Destruction of nitramines employing aqueous dispersions of metal powders. 1996, US5523517A.

[9] J.H. Kim, C. Jo, C.H. Lee and M.W. Byun, Reduction of carcinogenic N-nitrosamines and residual nitrite in model system sausage by irradiation, Journal of Food Science 67(4) (2006), 1370-1373. https://doi.org/10.1111/j.1365-2621.2002.tb10291.x

[10] G. Lunn, E.B. Sansone and L.K. Keefer, Reduction of nitrosamines with aqueous titanium trichloride: convenient preparation of aliphatic hydrazines, J. Org. Chem. 49(19) (1984), 3470-3473. https://doi.org/10.1021/jo00193a007

[11] A.K. Sikder and N. Sikder, A review of advanced high performance, insensitive and thermally stable energetic materials emerging for military and space applications, $J$. Hazardous Materials A 112 (2004), 1-15. https://doi.org/10.1016/j.jhazmat.2004.04.003

[12] V.T. Ramarkrishnan, M. Vedachalam and J.M. Boyer, 4,10-Dinitro-2,6,8,12-tetraoxa4,10-diazatetracyclo[5,5,0,05,903,11]dodecane, Heterocycles 31 (1990), 479-480. https://doi.org/10.3987/com-89-5192

[13] E.C. Koch, TEX - 4,10-Dinitro-2,6,8,12-tetraoxa-4,10-diazatetracyclo[5.5.0.0 $\left.0^{5,9} \cdot 0^{3,11}\right]$ dodecane - Review of a promising high density insensitive energetic material, Propellants Explos. Pyrotech. 40 (2015), 374-387.

https://doi.org/10.1002/prep.201400195

[14] Z.X. Li, Y.X. Ou and B.R. Chen, Synthesis of TEX from six kinds of different substituted piperazine, Hanneng Cailliao 9 (2001), 104-106.

[15] T.M. Klapötke and H.G. Ann, Estimation of the crystalline density of nitramine (NNO2 based) high energy density materials (HEDM), Propellants Explos. Pyrotech. 26 (2001), 221-224.

https://doi.org/10.1002/15214087(200112)26:5<221::AID-PREP221>3.0.CO;2-T

[16] S. Zeman, Relationship between detonation characteristics and 15N NMR chemical shifts of nitramines, J. Energetic Materials 17 (1999), 305-330. https://doi.org/10.1080/07370659908201793

[17] J.J.P. Stewart, Optimization of parameters for semiempirical methods I. Method, $J$. Comput. Chem. 10 (1989), 209-220. https://doi.org/10.1002/jcc.540100208

[18] J.J.P. Stewart, Optimization of parameters for semi empirical methods II. Application, $J$. Comput. Chem. 10 (1989), 221-264. https://doi.org/10.1002/jcc.540100209

[19] A.R. Leach, Molecular Modeling, Essex: Longman, 1997. 
[20] P. Fletcher, Practical Methods of Optimization, New York: Wiley, 1990.

[21] W. Kohn and L. Sham, Self-consistent equations including exchange and correlation effects, J. Phys. Rev. 140 (1965), 1133-1138. https://doi.org/10.1103/PhysRev.140.A1133

[22] R.G. Parr and W. Yang, Density Functional Theory of Atoms and Molecules, London: Oxford University Press, 1989.

[23] C.J. Cramer, Essentials of Computational Chemistry, Chichester, West Sussex: Wiley, 2004.

[24] A.D. Becke, Density-functional exchange-energy approximation with correct asymptotic behavior, Phys. Rev. A 38 (1988), 3098-3100. https://doi.org/10.1103/PhysRevA.38.3098

[25] S.H. Vosko, L. Wilk and M. Nusair, Accurate spin-dependent electron liquid correlation energies for local spin density calculations: a critical analysis, Can. J. Phys. 58 (1980), 1200-1211. https://doi.org/10.1139/p80-159

[26] C. Lee, W. Yang and R.G. Parr, Development of the Colle-Salvetti correlation-energy formula into a functional of the electron density, Phys. Rev. B 37 (1988), 785-789. https://doi.org/10.1103/PhysRevB.37.785

[27] SPARTAN 06, Wavefunction Inc., Irvine CA, USA, 2006.

[28] A.N. Nesmeyanov and N.A. Nesmeyanov, Fundamentals of Organic Chemistry, V.4, $2^{\text {nd }}$ ed., Moscow: Mir, 1981. 